\title{
THERAPEUTIC HYPOTHERMIA - A 24 HOUR CENTRALISED MODEL OF CARE
}

\author{
K. O'Reilly, J. Tooley, S. Winterbottom
}

NICU, St Michael's Hospital, Bristol, UK

Aims: Our neonatal network has provided a centralised service for therapeutic hypothermia (TH) since April 2008.The aim of this study was to evaluate the effectiveness of this service.

Methods: Data for all neonates referred for TH since April 2008 was retrospectively analysed.

Results: 92 referrals were received: 81 from our network and 11 from 3 other networks. No referral was refused. $46 \%$ of referrals occurred "out of hours" (20:00-08:00). 6 infants did not fulfil cooling criteria and 2 infants died prior to transfer. The remaining 84 infants were centralised within our network.

\begin{tabular}{|l|l||}
\hline & Median (range) \\
\hline Gestation (weeks) & $40(35-43)$ \\
\hline Birth weight (g) & $3238(1900-5250)$ \\
\hline Age at referral (mins) & $114(31-360)$ \\
\hline Age when team arrived at referring unit (mins) & $227(96-534)$ \\
\hline Temp when team arrived at referring unit $\left({ }^{\circ} \mathrm{C}\right)$ & $35.0(30-8-38)$ \\
\hline Stabilisation time (mins) & $140(45-355)$ \\
\hline Time from initial call to target temp $\left(33-34^{\circ} \mathrm{C}\right)($ mins) & $202(0-365)$ \\
\hline
\end{tabular}

[Infants centralised for therapeutic hypothermia]

Following 72 hours of TH, 72 infants were discharged home or back to their local unit (median stay 9 days). Short term outcome indicators were: death $14 \%$; severely abnormal MRI $23 \%$; mildly abnormal MRI $24 \%$; normal MRI 30\%.

Conclusions: Centralisation of $\mathrm{TH}$ allows equity of access to expertise whilst ensuring target core temperature is achieved within an acceptable time frame. A successful centralised TH service relies on early referral of eligible infants and a $24 \mathrm{hr}$ neonatal transfer service. 